Journal of Mathematics and Statistics 6 (4): 462-467, 2010

ISSN 1549-3644

(C) 2010 Science Publications

\title{
Two New Formulas for Generating Infinite Sets of Integer Numbers as a Sum of Two and Three Signed Cubic Numbers
}

\author{
Rand Alfaris and Hailiza Kamarulhaili \\ School of Mathematics Sciences, University Sains Malaysia, \\ Minden 11800, Penang, Malaysia
}

\begin{abstract}
Problem statement: Two new families are introduced for generating infinite sets of integers by the summation of two and three signed cubic numbers. Approach: These two families have reduced the number of representation of finite sets of integer as a sum of cubic numbers from four cubes to two and three signed cubes. Results: It is also shown that not all integers can be represented as a sum of two cubic numbers. The first family is called JR-2CN generates infinite set of integer numbers by the sum of two signed cubic numbers. The second family is named as JR-3CN generates infinite set of integer numbers by the sum of three signed cubic numbers. Conclusion/Recommendations: The integers in these two families have been generated by using quadratic polynomials and have been studied depending on the geometric analysis of the parabolas. Samples of integers generated by these two families are shown along with their properties and graphical behavior.
\end{abstract}

Key words: Cubic numbers, Integer representations, Quadratic polynomials

\section{INTRODUCTION}

Pollock conjectured that every number is the sum of at most 9 cubic numbers (Deshouillers et al., 2000). As part of Waring's problem (Conn and Vaserstein, 1994), it is known that every positive integer is a sum of at most 9 positive cubes and every sufficiently large integer is a sum of not more than 7 positive cubes (Bertault et al., 1999), with the exception of the two numbers 23 and 239 which can be represented as a sum of 9 cubes. Again related to the same problem, it has been proven that every integer is a sum of at most 5 signed cubes and it is believed that 5 can be reduced to 4 cubes (Davenport, 1939). It has also been conjectured in the same study, that the largest integer which can be represented as a sum of four nonnegative cubes is 7373170279850. It was a center of discussion in (Gardiner et al., 1964), on the numbers of the form $9 n \mp 4, n \in z$, where for this type of integers, the main concern was to find out whether or not this type of integer can be represented as a sum of four cubes. Until now, this question remains unanswered. Nevertheless, it has been proven in (Miller and Woollett, 1955) that there is no solution for integer $\mathrm{k}=$ $A^{3}+B^{3}+C^{3}$ when $k$ is the form $9 n \mp 4, n \in z$. With regard to this, we exclude this form of integers from our consideration. Aqel (2006) proves assertion value for each perspective is calculated and by using the algebraic summation method a conclusion can be determined.

\section{MATERIALS AND METHODS}

In this study, our interest is on the sum of three and two signed cubes. For three signed cubes, the previous studies have shown that for specific integers $\mathrm{N}$ with $\mathrm{N}<1000$ can be represented as a sum of three signed cubic numbers, (Ali et al., 2010; Heath-Brown et al., 1993; Dickson, 2007; Gardiner et al., 1964; Conway and Guy, 1996; Guy, 1994; Hardy and Wright, 2008). The research done in this study is an extension of the earlier results to the case for $\mathrm{N}$ exceeding 1000 and in fact, it covers infinite subset of integer numbers which is not in the form of $9 n \mp 4$.

There are three main results introduced in this study. First result proved that it is impossible to represent all the integer numbers as a sum of two signed cubes. Secondly, we introduced new family called JR$2 \mathrm{CN}$ which represents infinite subset of integers as a sum of two signed cubes. Third result introduced new family called JR-3CN which represents infinite sets of integers as a sum of three signed cubes. Properties and propositions are introduced with regards to these results.

Corresponding Author: Rand Alfaris, School of Mathematics Sciences, University Sains Malaysia,

Minden 11800, Penang, Malaysia 
This study organized as follows. The results and discussion are followed the introduction, where we introduce the two families for representing the infinite sets of integers as a sum of two and three signed cubes. Also, we introduce the properties of these two families. It ends with the conclusion and the references.

\section{RESULTS AND DISCUSSION}

The new families: As we have mentioned earlier, it has not been proven that every integer can be represented as a sum of four cubes. However, we can rest assured that not all integers can be represented as a sum of three signed cubes as the integers of the form $9 n \mp 4$ has no cubic solution. Here, we will prove that it is impossible to represent all the integers as a sum of two signed cubes. Therefore, representing every integer as a sum of 3 and 2 signed cubes will be meaningless. Instead, the question is: which integers can be represented as a sum of 3 and 2 signed cubes?

We already know as a result of the algebraic identity that the integers of the form:

$6 x$,

can be represented as the sum of four signed cubes:

$(\mathrm{x}+1)^{3}+(\mathrm{x}-1)^{3}-\mathrm{x}^{3}-\mathrm{x}^{3}$

Now, we introduce the integers that can be represented as a sum of two and three signed cubes. We will define the elements of the sets as JR-2CN and JR-3CN where we symbolized them as pairs.

The family JR-2CN: Before we introduce the new family, we will discuss some introductory results. The proof is actually an answer to the argument on representing all the integer numbers as a sum of two signed cubic numbers.

Proposition 1: The odd prime number cannot be represented as a sum of two signed cubes $A^{3} \mp B^{3}$ unless $\mathrm{A}-\mathrm{B}=1$.

Proof: Let $p$ be an odd prime number. We will assume that $\mathrm{p}$ can be represented as a sum of two signed cubes in all cases. Therefore:

$$
\mathrm{p}=\mathrm{A}^{3} \mp \mathrm{B}^{3}, \forall \mathrm{A}, \mathrm{B} \in \mathrm{Z}
$$

For the case $\mathrm{A}=\mathrm{B}$, we will get either $\mathrm{p}=0$ or $\mathrm{p}=$ $2 \mathrm{~A}^{3}$ and in either case $\mathrm{p}$ will not be an odd prime. Thus, we will drop this choice.

For the case $\mathrm{A} \neq \mathrm{B}$. By simplifying the equation above, we get:

$$
\mathrm{p}=(\mathrm{A} \mp \mathrm{B})\left(\mathrm{A}^{2} \mp \mathrm{AB}+\mathrm{B}^{2}\right), \forall \mathrm{A}, \mathrm{B} \in \mathrm{Z}
$$

This is a contradiction with the definition of prime numbers.

It is obvious that if $p=A^{3}-B^{3}=(A-B)\left(A^{2}+A B+\right.$ $B^{2}$ ) and $A-B=1$ then $p=A^{3}-B^{3}=A^{2}+A B+B^{2}$. Where there is no contradiction with the definition of the prime numbers.

This leaves us with the fact that we cannot represent the odd prime numbers as a sum of two signed cubes $\mathrm{A}^{3} \pm \mathrm{B}^{3}$, with the exception of the case $\mathrm{A}-\mathrm{B}=1$.

Proposition 2: Not all the integers can be represented as a sum of two cubic numbers.

Proof: By using proposition 2.1, take the odd prime number 3 as an example.

Now, we show which family of integers can be represented as a sum of two signed cubes.

Definition 1: JR-2CN is the family of integers that can be represented as a sum of two signed cubes $j^{3}+r^{3}$, where:

$\mathrm{j}=\mathrm{x}+\alpha, \mathrm{r}=\alpha-\mathrm{x} ; \forall \mathrm{x}, \alpha \in \mathrm{Z}$

Each member of JR-2CN is a form of an ordered pair whose components are the two signed cubes $(j, r)$.

The result of adding the two signed cubic numbers $\mathrm{j}^{3}+\mathrm{r}^{3}$ gives an integer number in the form:

$6 \alpha x^{2}+2 \alpha^{3}$

which is quadratic polynomial in $\mathrm{x}$ with the parameter $\alpha$.

We can put this set in a form of classes according to the integer parameter $\alpha$ such that:

$[\alpha]=\left\{6 \alpha x^{2}+2 \alpha^{3}: x \in Z\right\}$

The first few equations that belong to this family are:

$\pm 6 x^{2} \pm 2=(x \pm 1)^{3}+( \pm 1-x)^{3}, \alpha= \pm 1$
$\pm 12 x^{2} \pm 16=(x \pm 2)^{3}+( \pm 2-x)^{3}, \alpha= \pm 2$
$\pm 18 x^{2} \pm 54=(x \pm 3)^{3}+( \pm 3-x)^{3}, \alpha= \pm 3$

Table 1, shows a subset of cubes that represents the relating integers and its pairs $(\mathrm{j}, \mathrm{r})$ for Eq. 4-6.

We can see the graph in Fig. 1 below, that there is no intersection between these parabolas. We will show the proof and the advantages of this property in the discussion in the next section. 
J. Math. \& Stat., 6 (4): 462-467, 2010

Table 1: The integers generated by the family JR-3CN, the sum of two signed cubes $j^{3}+r^{3}=6 \alpha x^{2+} 2 \alpha^{3}$

\begin{tabular}{|c|c|c|c|c|c|c|}
\hline \multirow[b]{2}{*}{$\mathrm{x}$} & \multicolumn{2}{|l|}{$\alpha=1$} & \multicolumn{2}{|l|}{$\alpha=2$} & \multicolumn{2}{|l|}{$\alpha=3$} \\
\hline & $(j, r)$ & $6 x^{2}+2$ & $(j, r)$ & $12 x^{2}+16$ & $(j, r)$ & $18 x^{2}+54$ \\
\hline$\overline{-1}$ & $(0,2)$ & $8=0^{3}+2^{3}$ & $(1,3)$ & $28=1^{3}-3^{3}$ & $(2,4)$ & $72=2^{3}+4^{3}$ \\
\hline 0 & $(1,1)$ & $2=1^{3}+1^{3}$ & $(2,2)$ & $16=2^{3}-2^{3}$ & $(3,3)$ & $54=3^{3}+3^{3}$ \\
\hline 1 & $(2,0)$ & $8=2^{3}+0^{3}$ & $(3,1)$ & $28=3^{3}-1^{3}$ & $(4,2)$ & $72=4^{3}+2^{3}$ \\
\hline 2 & $(3,-1)$ & $26=3-1^{3}$ & $(4,0)$ & $46=4^{3}+0^{3}$ & $(5,1)$ & $126=5^{3}+1^{3}$ \\
\hline 3 & $(4,-2)$ & $56=4^{3}-2^{3}$ & $(5,1)$ & $124=5^{3}-1^{3}$ & $(6,0)$ & $126=6^{3}+0^{3}$ \\
\hline 4 & $(5,-3)$ & $98=5^{3}-3^{3}$ & $(6,-2)$ & $208=6^{3}-2^{3}$ & $(7,-1)$ & $342=7^{3}+1$ \\
\hline 5 & $(6,-4)$ & $152=6^{3}-4^{3}$ & $(7,-3)$ & $316=7^{3}-3^{3}$ & $(8,-2)$ & $504=8^{3}+2^{3}$ \\
\hline 6 & $(7,-5)$ & $218=7^{3}-5^{3}$ & $(8,-4)$ & $448=8^{3}-4^{3}$ & $(9,-3)$ & $702=9^{3}+3^{3}$ \\
\hline 7 & $(8,-6)$ & $396=8^{3}-6^{3}$ & $(9,-5)$ & $604=9^{3}-5^{3}$ & $(10,-4)$ & $936=10^{3}+4^{3}$ \\
\hline 8 & $\begin{array}{l}(0,-0) \\
(9,-7)\end{array}$ & $386=9^{3}-7^{3}$ & $(10,6)$ & $784=10^{3}-6^{3}$ & $(11,-5)$ & $1206=11^{3}+5^{3}$ \\
\hline 9 & $(10,-8)$ & $488=10^{3}-8^{3}$ & $(11,7)$ & $988=11^{3}-7^{3}$ & $(12,-6)$ & $1512=12^{3}+6^{3}$ \\
\hline 10 & $(11,-9)$ & $602=11^{3}-9^{3}$ & $(12,8)$ & $1216=12^{3}-8^{3}$ & $(13,-7)$ & $1854=13^{3}+7^{3}$ \\
\hline
\end{tabular}

Table 2: The integers generated by the family JR-3CN, the sum of three signed cubes $j^{3}+r^{3}-\alpha^{3}=3 \alpha^{2} r$

\begin{tabular}{llllll}
\hline$(\mathrm{j}, \mathrm{r})_{1}$ & $3 \mathrm{r}^{2}-3 \mathrm{r}$ & $(\mathrm{j}, \mathrm{r})_{2}$ & $63 \mathrm{r}^{2}-12 \mathrm{r}$ & $(\mathrm{j}, \mathrm{r})_{3}$ & $9 \mathrm{r}^{2}-27 \mathrm{r}$ \\
\hline$(0,1)$ & $0=0^{3}+1^{3}-1^{3}$ & $(0,2)$ & $0=0^{3}+2^{3}-2^{3}$ & $(0,3)$ & $0=0^{3}+3^{3}-3^{3}$ \\
$(1,0)$ & $0=1^{3}+0^{3}-1^{3}$ & $(1,1)$ & $-6=1^{3}+1^{3}-2^{3}$ & $(1,2)$ & $-18=1^{3}+2^{3}-3^{3}$ \\
$(2,1)$ & $6=2^{3}+1^{3}-1^{3}$ & $(2,0)$ & $0=3^{3}+0^{3}-2^{3}$ & $(3,0)$ & $18=2^{3}+1^{3}-3^{3}$ \\
$(3,-2)$ & $18=3^{3}+2^{3}-1^{3}$ & $(3,-1)$ & $18=3^{3}+1^{3}-2^{3}$ & $(4,-1)$ & $0=3^{3}+0^{3}-3^{3}$ \\
$(4,-3)$ & $36=4^{3}+3^{3}-1^{3}$ & $(4,-2)$ & $48=4^{3}+2^{3}-2^{3}$ & $36=4^{3}+1^{3}-3^{3}$ \\
$(5,-4)$ & $60=5^{3}+4^{3}-1^{3}$ & $(5,-3)$ & $90=5^{3}+3^{3}-2^{3}$ & $(5,-2)$ & $90=5^{3}+2^{3}-3^{3}$ \\
$(6,-5)$ & $90=6^{3}+5^{3}-1^{3}$ & $(6,-4)$ & $144=6^{3}+4^{3}-2^{3}$ & $(6,-3)$ & $162=6^{3}+3^{3}-3^{3}$ \\
$(7,-6)$ & $126=7^{3}+6^{3}-1^{3}$ & $(7,-5)$ & $210=71^{3}+5^{3}-2^{3}$ & $(7,-4)$ & $252=5^{3}+4^{3}-3^{3}$ \\
$(8,-7)$ & $168=8^{3}-7^{3}-1^{3}$ & $(8,-6)$ & $288=8^{3}+6^{3}-2^{3}$ & $(8,-5)$ & $360=8^{3}+5^{3}-3^{3}$ \\
$(9,-8)$ & $216=9^{3}+8^{3}-1^{3}$ & $(9,-7)$ & $378=9^{3}+7^{3}-2^{3}$ & $(9,-6)$ & $486=9^{3}+6^{3}-3^{3}$ \\
$(10,-9)$ & $270=10^{3}+9^{3}-1^{3}$ & $(10,-8)$ & $480=10^{3}+8^{3}-2^{3}$ & $(10,-7)$ & $630=10^{3}+7^{3}-3^{3}$ \\
\hline
\end{tabular}

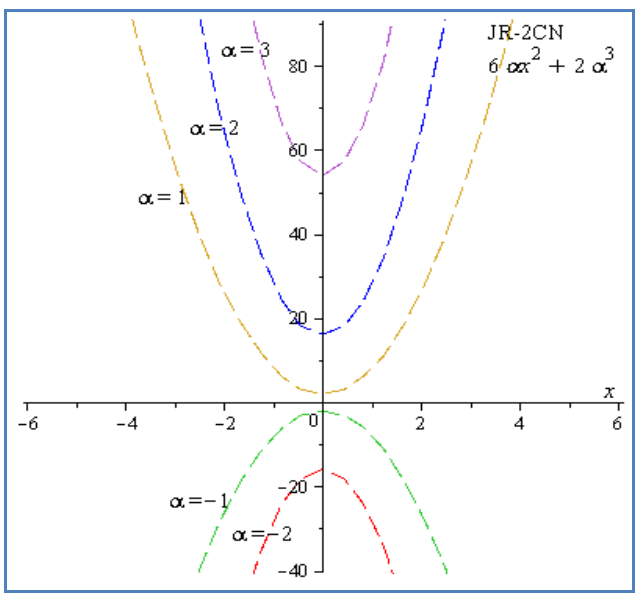

Fig. 1: Generating infinite sets of integers by using the family JR-2CN

\section{The family JR-3CN:}

Definition 2: JR-3CN is the family of all integers that can be represented as a sum of three signed cubes, each member of this family depends on the ordered pair $(\mathrm{j}, \mathrm{r})$ where $\mathrm{j}, \mathrm{r}$ and $\alpha$ are integers and $\alpha$ is the parameter, such that:

$$
j=\alpha-r
$$

The integers resulted from this family are of the sum of three signed cubes $j^{3}+r^{3}-a^{3}$ which gives the formula:

$$
3 \alpha r^{2}-3 \alpha^{2} r, \alpha \in Z
$$

Which is also quadratic polynomial in $\mathrm{r}$. The first few equations that belong to this family are:

$$
\begin{aligned}
& \pm 3 r^{2}-3 r=( \pm 1-r)^{3} r^{3}+1^{3}, \alpha= \pm 1 \\
& \pm 6 r^{2}-12 r=( \pm 2-r)^{3} r^{3}+2^{3}, \alpha= \pm 2 \\
& \pm 9 r^{2}-27 r=( \pm 3-r)^{3} r^{3}+3^{3}, \alpha= \pm 3
\end{aligned}
$$

The previous studies were on the natural numbers $\mathrm{n}<1000$. In this study, we have extended the result beyond this. 


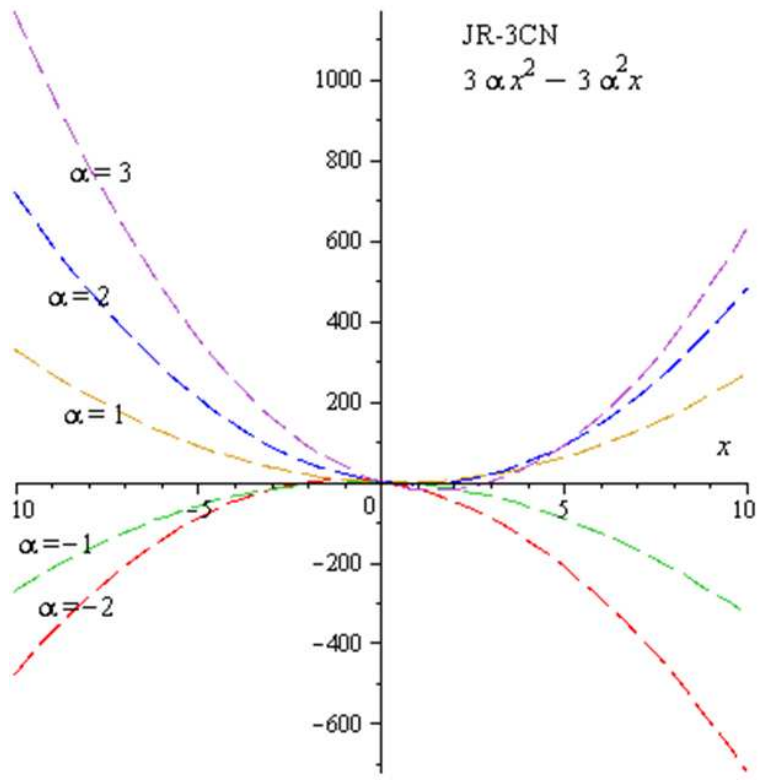

Fig. 2: Generating infinite sets of integers by using the formula JR-2CN

Figure 2 shows the behavior of the sum of three signed cubes.

Table 2, introduces the pairs $(\mathrm{j}, \mathrm{r})_{\alpha}$ that generate the integers family JR-3CN.

The previous studies were on the natural numbers $\mathrm{n}<1000$. In this study, we have extended the result beyond this.

\section{Properties of JR-2CN and JR-3CN:}

Type of numbers: It is easy to see that each integer in JR-2CN is a multiple of 2, while each integer in JR$3 \mathrm{CN}$ is a multiple of 3 .

Symmetric: (The symmetric property is related to the concept of the parabola. If the standard equation of the parabola is $y=\alpha x^{2}+b x+c$. Then the axis of symmetry is $\mathrm{x}=\frac{\mathrm{b}}{2 \alpha}$ (Akopyan and Zaslavskǐ̄, 2007):

- The parabolas formulate the family JR-2CN are symmetric around the vertical axis $\mathrm{x}=0$, therefore, each integer for the same parameter can be represented by using the pair $(\mathrm{j}, \mathrm{r})$ and the pair $(\mathrm{r}, \mathrm{j})$

- The parabolas that formulate the family JR-3CN are symmetric around the vertical line $x=\frac{b}{2} \alpha$ and therefore, each integer for the same parameter can be represented by using the pair $(\mathrm{j}, \mathrm{r})_{\alpha}$ and the pair $(r, j)_{\alpha}$

Intersections within the same family: We saw in Fig. 2 That there is an intersection within the family JR$3 \mathrm{CN}$. However, there is no intersection between any two different members in the family JR-2CN. We give the proof in the next two propositions.

Proposition 1: There are no integers in common for any two different members in JR-2CN.

Proof: For any two different members $6 \alpha x^{2}+2 \alpha^{2}$ and $6 \beta x^{2}+2 \beta^{3}$.

By solving them with respect to $\mathrm{x}$, we will find that:

$x^{2}=-\frac{1}{3}\left(\alpha^{2}+\alpha \beta+\beta^{2}\right)$

This cannot be a real number and hence integer number for any $\alpha, \beta \in \mathrm{z}$.

Proposition 2: There are intersections among the different members in JR-3CN.

Proof: for any two different members $6 \alpha r^{2}+2 \alpha^{2} r$ and $3 \beta x^{2}+3 \beta^{3}$ r.

By solving them with respect to $r$, we will find that:

$r^{2}-(\alpha+\beta) r=0$

And that will give the solution, when $r=0$ or $r=\alpha+\beta$.

For instance: we can represent 90 in two different ways as a sum of three signed cubic numbers by using the pair $(5,-3)_{2}$, or by using the pair $(5,-3)_{3}$. For this specific example we have, $\alpha=2$ and $\beta=3, r=5$, by using the symmetric property, $(5,-3)_{2}=(-3,5)_{2}$ and , $(5,-2)_{3}=(-2,5)_{3}$. It is obvious here that the intersection condition is verified by the two pairs $(-3$, $5)_{2}$ and $(-2,5)_{3}$.

Intersection between the two families for the same parameter: There is no intersection between the two families for the same parameter; that means, if the parameter is fixed, then the integers that can be represented as a sum of two cubes cannot represented as a sum of three cubes and vice versa. The proofs for these cases in next two propositions.

Proposition 3: There is no intersection between the families JR-2CN and JR-3CN for the same parameter. 


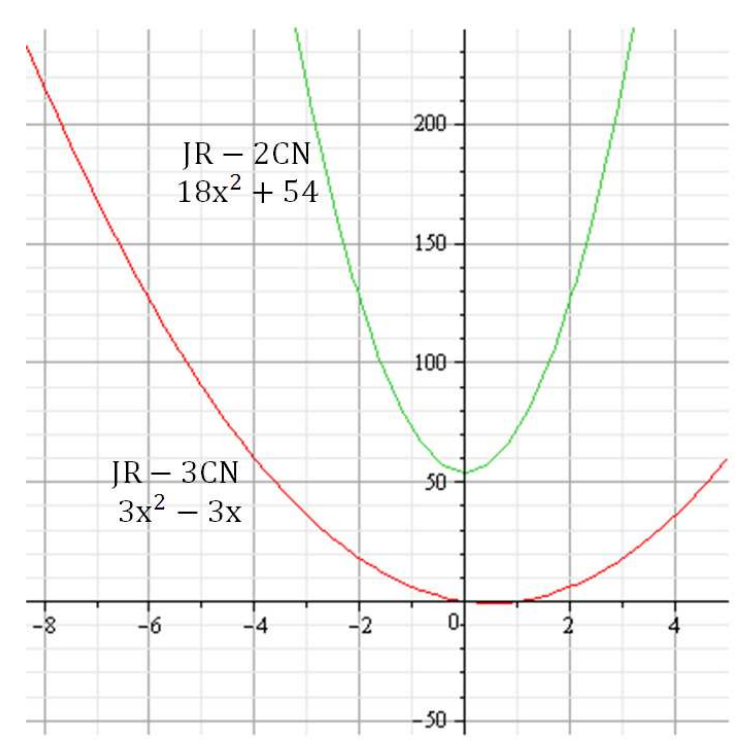

Fig. 3: There is no intersection between the parabola in JR-2CN for $\alpha=3, \mathrm{x}=3$ and the parabola in JR-3CN for $\alpha=1, \mathrm{x}=-8$

Proof: For the same parameter $\alpha$ we have $6 \alpha x^{2}+2 \alpha^{3}$ and $6 \alpha x^{2}-2 \alpha^{3} x$.

By solving them with respect to $\mathrm{x}$, we will find that:

$x=\frac{-3 \alpha^{2} \pm \sqrt{-15 \alpha^{4}}}{6 \alpha}$

which cannot be a real number and hence integer number for any $\alpha, \beta \in z$.

Intersection between the two families for different parameter: From the observations, we conjecture that there is no intersection between the two families for different parameters, in spite of this, we can find common integers among them. It means, there are some integers that can be represented as a sum of two cubes for a parameter, can also be represented as a sum of three cubes for the different parameter. For instance: Let us see the case when $\mathrm{a}=3$ and $\mathrm{x}=3$ in JR-2CN and when $\mathrm{a}=1$ and $\mathrm{x}=-8$ in JR-3CN. The integer 216 can be represented by using the pair $(6,0)$ and also can be represented by using the pair $(9,-8)$. Figure 3 shows that there is no intersection between the two parabolas.

\section{CONCLUSION}

We proved that not all the integers can be represented as a sum of two cubes. More specific, we proved that all the odd prime numbers cannot be represented as a sum of two signed cubes. We introduced two families to generate infinite sets of integers as a sum of three and two signed cubes. We also have discussed the properties of these two families.

Our next concern is on trying to answer the question: are there any other family apart from the two that are discussed in this study which can give completely different integers as a sum of three or two signed cubes. For two signed cubes, we are currently working on cubic polynomials of two variables and for three signed cubes, we are working with formulas that give quadratic and cubic polynomials. Also, we are working on slightly different formulas for the JR-3CN family that give cubic polynomial in two variables. The other concern is about the possibility of constructing Abelian groups and fields out of these families.

\section{REFERENCES}

Akopyan, A.V. andA.A. Zaslavskiǐ, 2007. Geometry of Conics, 26th Edn. American Mathematical Society (AMS). Ch1, pp: 19.

Ali, A.D., P.D. Swami and J. Singhai, 2010. Modified curvelet thresholding algorithm for image denoising. J. Comput. Sci., 6: 18-23. DOI: 10.3844/jcssp.2010.18.23.

Aqel, M.J., 2006. An approach for developing uncertain multi-perspective expert system using the algebraic sum method. Am. J. Applied Sci., 3: 1719-1721. DOI: 10.3844/.2006.1719.1721.

Bertault, F., O. Ramaré and P. Zimmermann, 1999. On Sums of Seven Cubes. Math. Comput. 68: 1303-1310.

Conn, B. and L. Vaserstein, 1994. On Sums of Three Integral Cubes. Contemp. Math., 166: 285-294.

Conway, J.H. and R.K. Guy, 1996. The Book of Numbers. 1st Edn., New York: Springer-Verlag, pp: 42-44. ISBN: 0-387-97993-X

Davenport, H., 1939.On Waring,s Problem for Cubes. Acta Math., 71: 123-143.

Deshouillers, J.M., F. Hennecart and B. Landreau, 2000. 7373170279 850. Math. Comput., 69: 421-439.

Dickson, L.E., 2007. History of the Theory of Numbers, Diophantine Analysis. 2nd Edn., New York, Dover, pp: 832. ISBN: 0486442330

Gardiner, V. L., R. B. Lazarus and P.R. Stein, 1964. Solutions of the Diophantine Equation $\mathrm{x}^{3}+\mathrm{y}^{3}$ $=\mathrm{z}^{3}$-d. Math. Comput., 18: 408-413.

Guy, R.K., 1994. Sum of Four Cubes. D5 in Unsolved Problems in Number Theory. 2nd Edn. New York: Springer-Verlag, pp: 151-152. 
Hardy, G.H. and E.M. Wright, 2008. Representation by Cubes and Higher Powers. Ch. 21 in An Introduction to the Theory of Numbers. 6th Edn. Oxford, England: Clarendon Press, pp: 317-339.

Heath-Brown, D. R., W.M. Lioen and H.J.J. Te Riele, 1993. On Solving the Diophantine Equation $x^{3}+y^{3}$ $+\mathrm{z}^{3}=\mathrm{k}$ on a Vector Computer. Math. Comput., 61: 235-244.
Miller, J.C.P. and M. F. C. Woollett, 1955. Solutions of the Diophantine Equation $\mathrm{x}^{3}+\mathrm{y}^{3}+\mathrm{z}^{3}=\mathrm{k}$. J. London Math. Soc., 30: 101-110. 